\title{
Hypercyclic sequences of operators
}

\author{
by \\ Fernando León-SaAvedra (Cádiz) and Vladimír Müller (Praha)
}

\begin{abstract}
A sequence $\left(T_{n}\right)$ of bounded linear operators between Banach spaces $X, Y$ is said to be hypercyclic if there exists a vector $x \in X$ such that the orbit $\left\{T_{n} x\right\}$ is dense in $Y$. The paper gives a survey of various conditions that imply the hypercyclicity of $\left(T_{n}\right)$ and studies relations among them. The particular case of $X=Y$ and mutually commuting operators $T_{n}$ is analyzed. This includes the most interesting cases $\left(T^{n}\right)$ and $\left(\lambda_{n} T^{n}\right)$ where $T$ is a fixed operator and $\lambda_{n}$ are complex numbers. We also study when a sequence of operators has a large (either dense or closed infinite-dimensional) manifold consisting of hypercyclic vectors.
\end{abstract}

I. Introduction. Let $X$ and $Y$ be separable Banach spaces. Denote by $B(X, Y)$ the set of all bounded linear operators from $X$ to $Y$. Let $\left(T_{n}\right) \subset$ $B(X, Y)$ be a sequence of operators. A vector $x \in X$ is called hypercyclic for $\left(T_{n}\right)$ if the set $\left\{T_{n} x\right\}$ is dense in $Y$. The sequence $\left(T_{n}\right)$ is called hypercyclic if there is at least one vector hypercyclic for $\left(T_{n}\right)$. We say that an operator $T: X \rightarrow X$ is hypercyclic if the sequence $\left(T^{n}\right)$ of its iterates is hypercyclic.

Similarly, an operator $T$ is said to be supercyclic if there exists a vector $x \in X$ such that the set $\left\{\lambda T^{n} x: \lambda \in \mathbb{C}, n \in \mathbb{N}\right\}$ is dense; the vector $x$ with this property is called supercyclic for $T$.

Usually it is not easy to verify whether a sequence $\left(T_{n}\right)$ is hypercyclic or not. There are many criteria that have been studied by a number of authors that imply the hypercyclicity of $\left(T_{n}\right)$ (see e.g. $[\mathrm{K}],[\mathrm{GS}],[\mathrm{BG}],[\mathrm{BP}]$ ). In the second section we give a survey of various conditions implying the hypercyclicity and study relations among them. A number of illustrative examples are given.

The third section concentrates on the situation when $Y=X$ and the operators $T_{n}: X \rightarrow X$ are mutually commuting. The relations among vari-

2000 Mathematics Subject Classification: Primary 47A16; Secondary 47A53.

Key words and phrases: hypercyclic sequences, hypercyclic vectors, supercyclic vectors, hypercyclic subspaces, essential minimum modulus.

The first-named author partially supported by Junta de Andalucía FQM-257.

The second-named author supported by the grant No. 201/03/0041 of GA ČR. 
ous conditions are much simpler in this case. The following section studies the case when $T_{n}=S_{1} \cdots S_{n}$ where $S_{j}: X \rightarrow X$ are mutually commuting. This includes the most interesting cases $\left(T^{n}\right)$ and $\left(\lambda_{n} T^{n}\right)$ where $T$ is a fixed operator and $\lambda_{n}$ complex numbers.

Sequences of operators with "many hypercyclic vectors" are very important in hypercyclicity theory. The interest in them (especially in the cases of $\left(T^{n}\right)$ and $\left.\left(\lambda_{n} T^{n}\right)\right)$ arises from the invariant subspace/subset problem.

There are two research lines in the literature. The first one, which was initiated by B. Beauzamy [Bea] and continued in [G], [GS], [He], [Bo], [B], $[\mathrm{BC}]$ and recently [Gri], studies the existence of dense manifolds consisting of hypercyclic vectors. The second, more recent, line studies the existence of closed infinite-dimensional subspaces all of whose nonzero elements are hypercyclic; see $[\mathrm{Mo}],[\mathrm{LMo}],[\mathrm{GLM}]$ and recently $[\mathrm{BMP}]$. The questions of this type are studied in Section V below.

II. Hypercyclicity of sequences of operators. Let $X, Y$ be separable Banach spaces and let $\left(T_{n}\right) \subset B(X, Y)$ be a sequence of operators. It is well known that the set of all hypercyclic vectors for $\left(T_{n}\right)$ is a $G_{\delta}$ set. Indeed, $x \in X$ is hypercyclic for $\left(T_{n}\right)$ if and only if $x \in \bigcap_{U} \bigcup_{n \in \mathbb{N}} T_{n}^{-1} U$, where $U$ runs over a countable base of open subsets of $Y$; it is clear that $\bigcup_{n \in \mathbb{N}} T_{n}^{-1} U$ is open for each $U$.

Lemma 1 ([GS]). Let $\left(T_{n}\right) \subset B(X, Y)$ be a sequence of operators. The following conditions are equivalent:

(i) $\left(T_{n}\right)$ has a dense subset of hypercyclic vectors;

(ii) the set of all hypercyclic vectors for $\left(T_{n}\right)$ is residual (i.e., its complement is of the first category);

(iii) for all nonempty open subsets $U \subset X, V \subset Y$ there exists $n \in \mathbb{N}$ such that $T_{n} U \cap V \neq \emptyset$;

(iv) for all $x \in X, y \in Y$ and $\varepsilon>0$ there exist $n \in \mathbb{N}$ and $u \in X$ such that $\|u-x\|<\varepsilon$ and $\left\|T_{n} u-y\right\|<\varepsilon$.

Denote by $B_{X}$ the closed unit ball in a Banach space $X$. The most practical criteria of hypercyclicity are the following two:

Definition 2. We say that a sequence $\left(T_{n}\right) \subset B(X, Y)$ satisfies condition $(\mathrm{C})$ if there exist an increasing sequence $\left(n_{k}\right)$ of positive integers and a dense subset $X_{0} \subset X$ such that

(i) $\lim _{k \rightarrow \infty} T_{n_{k}} x=0\left(x \in X_{0}\right)$;

(ii) $\bigcup_{k} T_{n_{k}} B_{X}$ is dense in $Y$.

The second condition is similar: 
Definition 3. We say that a sequence $\left(T_{n}\right) \subset B(X, Y)$ satisfies condition $\left(\mathrm{C}_{\text {fin }}\right)$ if there exist an increasing sequence $\left(n_{k}\right)$ of positive integers and a dense subset $X_{0} \subset X$ such that

(i) $\lim _{k \rightarrow \infty} T_{n_{k}} x=0\left(x \in X_{0}\right)$;

(ii) $\bigcup_{k}(\underbrace{T_{n_{k}} B_{X} \oplus \cdots \oplus T_{n_{k}} B_{X}}_{j})$ is dense in $\underbrace{Y \oplus \cdots \oplus Y}_{j}$ for all $j \in \mathbb{N}$.

Clearly, condition $\left(\mathrm{C}_{\mathrm{fin}}\right)$ implies $(\mathrm{C})$. Condition $(\mathrm{C})$ is the weakest known property which can be practically used to show the hypercyclicity of a sequence $\left(T_{n}\right)$. Moreover, it implies the existence of a dense (and hence residual) set of hypercyclic vectors. Furthermore, under a reasonable additional condition it implies that there is a closed infinite-dimensional subspace of hypercyclic vectors.

Condition $\left(\mathrm{C}_{\mathrm{fin}}\right)$ has a number of equivalent formulations and it implies that there is a dense linear subspace consisting of hypercyclic vectors. The existence of subspaces consisting of hypercyclic vectors will be studied in Section V.

Theorem 4. Let $\left(T_{n}\right) \subset B(X, Y)$ be a sequence of operators. The following conditions are equivalent:

(i) $\left(T_{n}\right)$ satisfies condition $(\mathrm{C})$;

(ii) for all $j \in \mathbb{N}$ and nonempty open subsets $U_{0}, U_{1}, \ldots, U_{j} \subset X$ and $V_{0}, V \subset Y$ such that $U_{0}$ and $V_{0}$ contain the origins of $X$ and $Y$, respectively, there exists $n \in \mathbb{N}$ such that $T_{n} U_{i} \cap V_{0} \neq \emptyset(i=1, \ldots, j)$ and $T_{n} U_{0} \cap V \neq \emptyset$.

In particular, if $\left(T_{n}\right)$ satisfies $(\mathrm{C})$ then there is a dense set of hypercyclic vectors for $\left(T_{n}\right)$.

Proof. (i) $\Rightarrow$ (ii). Clear.

(ii) $\Rightarrow$ (i). Let $\left(x_{n}\right) \subset X$ and $\left(y_{n}\right) \subset Y$ be dense sequences. Set $u_{i, i}=x_{i}$ $(i \in \mathbb{N})$. By induction on $k$ we construct an increasing sequence $\left(n_{k}\right)$ and vectors $u_{i, k} \in X(i=1, \ldots, k-1)$ and $v_{k} \in B_{X}$ such that

$$
\begin{aligned}
\left\|T_{n_{k}} v_{k}-y_{k}\right\| & <2^{-k}, \quad\left\|T_{n_{k}} u_{i, k}\right\|<2^{-k}, \\
\left\|u_{i, k}-u_{i, k-1}\right\| & <\frac{1}{2^{k} \max \left\{1,\left\|T_{n_{1}}\right\|, \ldots,\left\|T_{n_{k-1}}\right\|\right\}},
\end{aligned}
$$

for all $i, k$ with $1 \leq i<k$. For each $i$ the sequence $\left(u_{i, k}\right)_{k}$ is Cauchy. Let $u_{i}$ be its limit. Then

$$
\left\|x_{i}-u_{i}\right\| \leq \sum_{k=i+1}^{\infty}\left\|u_{i, k}-u_{i, k-1}\right\| \leq \sum_{k=i+1}^{\infty} \frac{1}{2^{k}}=\frac{1}{2^{i}} .
$$

Therefore $\left(u_{i}\right)$ is dense in $X$. 
Clearly the sequence $\left(T_{n_{k}} v_{k}\right)$ is dense, and so $\overline{\bigcup_{k} T_{n_{k}} B_{X}}=Y$. Further,

$$
\begin{aligned}
\lim _{k \rightarrow \infty}\left\|T_{n_{k}} u_{i}\right\| & \leq \lim _{k \rightarrow \infty}\left(\left\|T_{n_{k}} u_{i, k}\right\|+\sum_{j=k}^{\infty}\left\|T_{n_{k}}\right\| \cdot\left\|u_{i, j+1}-u_{i, j}\right\|\right) \\
& \leq \lim _{k \rightarrow \infty}\left(\frac{1}{2^{k}}+\sum_{j=k}^{\infty} \frac{1}{2^{j+1}}\right)=\lim _{k \rightarrow \infty} \frac{1}{2^{k-1}}=0
\end{aligned}
$$

for each $i$. Thus $\left(T_{n}\right)$ satisfies (C).

To show that condition (C) implies the existence of a dense subset of hypercyclic vectors we use Lemma 1 . Let $x \in X, y \in Y$ and $\varepsilon>0$. By (ii), there are $x_{0}, x_{1} \in X$ and $n \in \mathbb{N}$ such that $\left\|x_{0}\right\|<\varepsilon / 2,\left\|T_{n} x_{0}-y\right\|<$ $\varepsilon / 2,\left\|x-x_{1}\right\|<\varepsilon / 2$ and $\left\|T_{n} x_{1}\right\|<\varepsilon / 2$. Then $\left\|\left(x_{0}+x_{1}\right)-x\right\|<\varepsilon$ and $\left\|T_{n}\left(x_{0}+x_{1}\right)-y\right\|<\varepsilon$. By Lemma $1,\left(T_{n}\right)$ has a dense subset of hypercyclic vectors.

TheOREm 5. Let $\left(T_{n}\right) \subset B(X, Y)$ be a sequence of operators. The following conditions are equivalent:

(i) $\left(T_{n}\right)$ satisfies condition $\left(\mathrm{C}_{\text {fin }}\right)$;

(ii) $(\underbrace{T_{n} \oplus \cdots \oplus T_{n}}_{j})$ satisfies condition $(\mathrm{C})$ for all $j \in \mathbb{N}$;

(iii) $(\underbrace{T_{n} \oplus \cdots \oplus T_{n}}_{j})$ has a dense subset of hypercyclic vectors for all $j \in \mathbb{N}$

(iv) for all $j \in \mathbb{N}$ and all nonempty open subsets $U_{1}, \ldots, U_{j} \subset X$ and $V_{1}, \ldots, V_{j} \subset Y$ there is an $n \in \mathbb{N}$ such that $T_{n} U_{i} \cap V_{i} \neq \emptyset$ $(i=1, \ldots, j)$;

(v) there is a subsequence $\left(T_{n_{k}}\right)$ such that each of its subsubsequences has a dense set of hypercyclic vectors;

(vi) there are dense subsets $X_{0} \subset X$ and $Y_{0} \subset Y$, an increasing sequence $\left(n_{k}\right) \subset \mathbb{N}$ and mappings $S_{i}: Y_{0} \rightarrow X(i \in \mathbb{N})$ such that

$$
\begin{aligned}
T_{n_{k}} x \rightarrow 0 & \quad\left(x \in X_{0}\right), \\
S_{k} y \rightarrow 0 & \left(y \in Y_{0}\right), \\
T_{n_{k}} S_{k} y \rightarrow y & \left(y \in Y_{0}\right) ;
\end{aligned}
$$

(vii) for each Banach space $Z$ the sequence of operators $L_{T_{n}}: \overline{F(Z, X)}$ $\rightarrow \overline{F(Z, Y)}$ defined by $L_{T_{n}} S=T_{n} S(S \in \overline{F(Z, X)})$ has a dense set of hypercyclic vectors; here $F(Z, X)$ denotes the set of all finite rank operators from $Z$ to $X$;

(viii) for each Banach space $Z$ the sequence $\left(L_{T_{n}}\right)$ satisfies condition $(\mathrm{C})$.

Proof. The equivalences (vi) $\Leftrightarrow(\mathrm{v}) \Leftrightarrow$ (iii) were proved in [BG]. The implications (i) $\Rightarrow$ (ii) and (vi) $\Rightarrow$ (i) are obvious. The equivalence (iii) $\Leftrightarrow$ (iv) follows 
from Lemma 1 and the implications (viii) $\Rightarrow$ (vii) and (ii) $\Rightarrow$ (iii) follow from Theorem 4.

(i) $\Rightarrow$ (viii). Let $X_{0}$ be a dense subset of $X$ and let $\left(n_{k}\right)$ satisfy $T_{n_{k}} x \rightarrow 0$ $\left(x \in X_{0}\right)$ and $\overline{U\left(T_{n_{k}} B_{X} \oplus \cdots \oplus T_{n_{k}} B_{X}\right)}=Y \oplus \cdots \oplus Y$.

Let $\mathcal{M} \subset B(Z, X)$ be the set of all finite rank operators with range included in the linear space generated by $X_{0}$. Clearly $\mathcal{M}$ is dense in $\overline{F(Z, X)}$. For $G \in \mathcal{M}$ we have $\lim _{k} L_{T_{n_{k}}} G=\lim _{k} T_{n_{k}} G=0$.

Let $F \in F(Z, Y)$ and $\varepsilon>0$. We can express $F=\sum_{i=1}^{j} z_{i}^{*} \otimes y_{i}$ for some $y_{i} \in Y$ and $z_{i}^{*} \in Z^{*}$. Since $\left(T_{n}\right)$ satisfies condition $\left(\mathrm{C}_{\text {fin }}\right)$, there are vectors $u_{i} \in X(i=1, \ldots, j)$ and $k \in \mathbb{N}$ such that

$$
\left\|T_{n_{k}} u_{i}-y_{i}\right\|<\frac{\varepsilon}{j \max \left\{\left\|z_{1}^{*}\right\|, \ldots\left\|z_{j}^{*}\right\|\right\}}, \quad\left\|u_{i}\right\| \leq \frac{1}{j \max \left\{\left\|z_{1}^{*}\right\|, \ldots,\left\|z_{j}^{*}\right\|\right\}} .
$$

Set $F_{0}=\sum_{i=1}^{j} z_{i}^{*} \otimes u_{i} \in F(Z, X)$. Then $\left\|F_{0}\right\| \leq \sum_{i=1}^{j}\left\|z_{i}^{*}\right\| \cdot\left\|u_{i}\right\| \leq 1$ and

$$
\begin{aligned}
\left\|L_{T_{n_{k}}} F_{0}-F\right\| & =\left\|T_{n_{k}} F_{0}-F\right\|=\left\|\sum_{i=1}^{j} z_{i}^{*} \otimes T_{n_{k}} u_{i}-\sum_{i=1}^{j} z_{i}^{*} \otimes y_{i}\right\| \\
& =\left\|\sum_{i=1}^{j} z_{i}^{*} \otimes\left(T_{n_{k}} u_{i}-y_{i}\right)\right\| \leq \sum_{i=1}^{j}\left\|z_{i}^{*}\right\| \cdot\left\|T_{n_{k}} u_{i}-y_{i}\right\|<\varepsilon .
\end{aligned}
$$

(vii) $\Rightarrow$ (iii). Let $j \in \mathbb{N}$ and let $Z$ be a $j$-dimensional Banach space. Then $\overline{F(Z, X)}$ is isomorphic to $\underbrace{X \oplus \cdots \oplus X}_{j}$ and $\overline{F(Z, Y)}$ to $\underbrace{Y \oplus \cdots \oplus Y}_{j}$. In the same way $L_{T_{n}}$ can be identified with $T_{n} \oplus \cdots \oplus T_{n}$.

For completeness we also mention other conditions that have been studied in the literature, for instance, in [BG, Theorem 2.2] (see also [BP, Remark $2.6(3)]$ in the case of commuting operators with dense range). In the diagram below we show the relations among them. The abbreviations there mean:

(HC) (Hypercyclicity criterion) There exist dense subsets $X_{0} \subset X$ and $Y_{0} \subset Y$, an increasing sequence $\left(n_{k}\right) \subset \mathbb{N}$ and mappings $S_{k}: Y_{0} \rightarrow X$ such that

$$
\begin{aligned}
T_{n_{k}} x \rightarrow 0 & \left(x \in X_{0}\right), \\
S_{k} y \rightarrow 0 & \left(y \in Y_{0}\right), \\
T_{n_{k}} S_{k} y=y & \left(y \in Y_{0}, k \in \mathbb{N}\right) .
\end{aligned}
$$

(hc) $\left(T_{n}\right)$ is hypercyclic.

(dense hc) $\left(T_{n}\right)$ has a dense set of hypercyclic vectors.

(4 nbhd) (4 neighbourhoods condition) for all nonempty open subsets $U, U_{0} \subset X$ and $V, V_{0} \subset Y$ such that $U_{0}$ and $V_{0}$ contain the origins in $X$ and $Y$, respectively, there exists $n \in \mathbb{N}$ such 
that $T_{n} U \cap V_{0} \neq \emptyset$ and $T_{n} U_{0} \cap V_{n} \neq \emptyset \quad$ (when $X=Y$ this condition reduces to "the three open sets condition", which was introduced in [GS, Section III]).

(her hc) (Hereditarily hypercyclic) There is a subsequence $\left(T_{n_{k}}\right)$ such that each of its subsubsequences is hypercyclic.

The relations among these conditions are given in the following diagram:

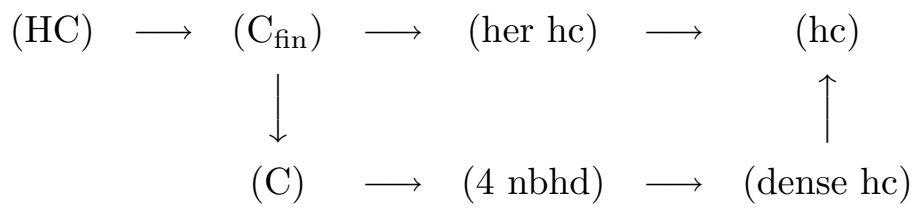

Moreover, there are no other implications among the conditions in question.

The implications $(\mathrm{HC}) \rightarrow\left(\mathrm{C}_{\mathrm{fin}}\right)$ and $\left(\mathrm{C}_{\mathrm{fin}}\right) \rightarrow($ her hc) were proved in Theorem 5 , the implication $(\mathrm{C}) \rightarrow(4 \mathrm{nbhd})$ in Theorem 4 . For the implication $(4 \mathrm{nbhd}) \rightarrow$ (dense hc) see Proposition 6 below.

The remaining implications are trivial.

The negative results follow from the examples below. Note that it is sufficient to show $\left(\mathrm{C}_{\text {fin }}\right) \not \rightarrow(\mathrm{HC}),(\mathrm{C}) \not \rightarrow($ her hc), (her hc) $\nrightarrow$ (dense hc) and (dense hc) $\nrightarrow$ (C).

Proposition 6 (cf. [GS]). If $\left(T_{n}\right) \subset B(X, Y)$ satisfies (4 nbhd) then there is a dense subset of hypercyclic vectors for $\left(T_{n}\right)$.

Proof. The result was essentially proved already in the proof of Theorem 4. Let $x \in X, y \in Y$ and $\varepsilon>0$. Then there are $n \in \mathbb{N}, u \in X$ and $v \in Y$ such that $\|u-x\|<\varepsilon / 2,\left\|T_{n} u\right\|<\varepsilon / 2,\|v\|<\varepsilon / 2$ and $\left\|T_{n} u-y\right\|<\varepsilon / 2$. Set $x^{\prime}=u+v$. Then $\left\|x-x^{\prime}\right\| \leq\|x-u\|+\|v\|<\varepsilon$ and $\left\|T_{n} x^{\prime}-y\right\|<\left\|T_{n} v-y\right\|+\left\|T_{n} u\right\|<\varepsilon$. By Lemma 1, this implies that $\left(T_{n}\right)$ has a dense subset of hypercyclic vectors.

EXAmple 7. Let $X$ be a Hilbert space with an orthonormal basis $\left\{e_{F}\right.$ : $F \subset \mathbb{N}$, card $F<\infty\}$. Let $Y=X$ and let the operators $T_{n}: X \rightarrow X$ be defined by

$$
T_{n} e_{F}= \begin{cases}n e_{F \backslash\{n\}} & (n \in F), \\ 0 & (n \notin F) .\end{cases}
$$

It is easy to verify that the sequence $\left(T_{n}\right)$ satisfies condition $\left(\mathrm{C}_{\text {fin }}\right)$ for the dense subspace $X_{0} \subset X$ generated by the vectors $\left\{e_{F}: F \subset \mathbb{N}\right\}$. However, $\left(T_{n}\right)$ does not satisfy (HC) since the operators $T_{n}$ have nondense ranges, $T_{n} X=\bigvee\left\{e_{F}: n \notin F\right\}$.

Note that the operators $T_{n}$ are even commuting.

Example 8. Let $X$ and $T_{n}: X \rightarrow X$ be as in the previous example. Note that $e_{\{n\}} \perp T_{n} X$ for each $n$. Consider the operators $S_{n}: X \oplus \mathbb{C} \rightarrow X$ 
defined by

$$
S_{n}(x \oplus \lambda)=T_{n} x+\lambda e_{\{n\}} \quad(x \in X, \lambda \in \mathbb{C}) .
$$

Since the operators $\left(T_{n}\right)$ satisfy $\left(\mathrm{C}_{\text {fin }}\right)$ and hence are hereditarily hypercyclic, it is easy to see that the sequence $\left(S_{n}\right)$ is also hereditarily hypercyclic. On the other hand, the set of all vectors hypercyclic for $\left(S_{n}\right)$ is not dense. Indeed, let $x \in X, \lambda \in \mathbb{C}, \lambda \neq 0$. Then

$$
\left\|S_{n}(x \oplus \lambda)\right\|=\left\|T_{n} x+\lambda e_{\{n\}}\right\| \geq|\lambda|>0,
$$

and so $x \oplus \lambda$ is not hypercyclic. This shows that (her hc) $\nrightarrow$ (dense hc).

EXAMPLE 9. Let $X$ be a separable Hilbert space with an orthonormal basis $\left\{e_{1}, e_{2}, \ldots\right\}$. Let $Y=\mathbb{C}^{2}$ and $\left(y_{n}\right)$ be a dense sequence of elements of $Y$. Define $T_{n}: X \rightarrow Y$ by $T_{n} e_{n}=y_{n}$ and $T_{n} e_{i}=0(i \neq n)$. Clearly $T_{n} x \rightarrow 0$ for each $x$ that is a finite linear combination of the vectors $e_{i}(i \in \mathbb{N})$. Further $\overline{\bigcup_{n} T_{n} B_{X}} \supset\left\{y_{n}: n \in \mathbb{N}\right\}^{-}=Y$. Thus $\left(T_{n}\right)$ satisfies condition (C).

On the other hand, let $\left(n_{k}\right)$ be any increasing sequence of positive integers such that $\left(T_{n_{k}}\right)$ is hypercyclic. Let $U \subset Y$ be a nonempty open set such that $\bar{U} \neq Y$ and $\mathbb{C} \cdot U \subset U$. Choose a subsequence of those indices $n_{k}$ for which $y_{n_{k}} \in U$. For such an $n_{k}$ we have $T_{n_{k}} X=\mathbb{C} \cdot y_{n_{k}} \subset U$, and so $\left(T_{n}\right)$ is not hereditarily hypercyclic. Consequently, $(\mathrm{C}) \nrightarrow \rightarrow$ (her hc).

Example 10. Let $\operatorname{dim} X=1$ (i.e., $X=\mathbb{C}$ ) and let $Y$ be a separable Hilbert space. Let $\left(y_{n}\right)$ be a dense sequence in $Y$. Define $T_{n}: X \rightarrow Y$ by $T_{n}(\lambda)=\lambda y_{n}$. Clearly each nonzero $\lambda \in X$ is hypercyclic for $\left(T_{n}\right)$. It is easy to see that $\left(T_{n}\right)$ does not satisfy condition (4 nbhd). Indeed, consider the neighbourhoods $U=\{z \in \mathbb{C}:|z|>2\}, V_{0}=\{y \in Y:\|y\|<1\}$, $U_{0}=\{z \in \mathbb{C}:|z|<1\}$ and $V=\{y \in Y:\|y\|>2\}$. Thus (dense hc) t $\rightarrow(4$ nbhd $)$.

EXAmPLe 11. Let $X=\mathbb{C}^{2}$ and $\operatorname{dim} Y=\infty$. Let $\left(y_{n}\right)$ be a dense sequence in $Y$ and $\left(x_{n}\right)$ dense in $X$. For each $n$ find $u_{n} \in X$ linearly independent of $x_{n}$ such that $\left\|u_{n}\right\|=1 / n$. For $m, n \in \mathbb{N}$ define $T_{m, n}: X \rightarrow Y$ by $T_{m, n} x_{n}=0$ and $T_{m, n} u_{n}=y_{m}$. Then $\left(T_{m, n}\right)$ is a countable set of operators satisfying condition (4 nbhd).

Let $\left(T_{m_{k}, n_{k}}\right)$ be any subsequence such that $T_{m_{k}, n_{k}} x \rightarrow 0$ for all $x$ in a dense subset of $X$. Then $T_{m_{k}, n_{k}} \rightarrow 0$ in the strong operator topology, and therefore this subsequence is bounded by the Banach-Steinhaus theorem. Thus $\left(T_{m, n}\right)$ does not satisfy $(\mathrm{C})$. Hence $(4 \mathrm{nbhd}) \nrightarrow(\mathrm{C})$.

III. Sequences of commuting operators. In this section we assume that $Y=X$ and $\left(T_{n}\right) \subset B(X)$ is a sequence of mutually commuting operators. The situation is much simpler in this case.

TheOREM 12. Let $\left(T_{n}\right) \subset B(X)$ be a sequence of mutually commuting operators. The following conditions are equivalent: 
(i) $\left(T_{n}\right)$ satisfies condition $(\mathrm{C})$;

(ii) $\left(T_{n}\right)$ satisfies condition $\left(\mathrm{C}_{\text {fin }}\right)$;

(iii) $\left(T_{n}\right)$ is hereditarily hypercyclic;

(iv) $\left(T_{n}\right)$ satisfies (4 nbhd); in fact in this case the 4 neighbourhoods condition reduces to the "3 neighbourhoods condition": for all nonempty open subsets $U, V, W \subset X$ with $0 \in W$ there exists $n$ such that $T_{n} U \cap W \neq \emptyset$ and $T_{n} W \cap V \neq \emptyset$.

Proof. (i) $\Rightarrow$ (ii). Let $X_{0} \subset X$ be a dense subset and $\left(n_{k}\right) \subset \mathbb{N}$ an increasing sequence such that $T_{n_{k}} x \rightarrow 0\left(x \in X_{0}\right)$ and $\overline{\bigcup_{k} T_{n_{k}} B_{x}}=X$.

By Theorem $4,\left(T_{n_{k}}\right)$ is hypercyclic. Let $x \in X$ be a hypercyclic vector for $\left(T_{n_{k}}\right)$.

Let $y_{1}, \ldots, y_{r} \in X$ and $\varepsilon>0$. Since $x$ is hypercyclic, there are $k_{1}, \ldots, k_{r}$ such that $\left\|T_{n_{k_{i}}} x-y_{i}\right\|<\varepsilon / 2(i=1, \ldots, r)$. Further, there are $u \in X$, $\|u\| \leq \max \left\{\left\|T_{n_{k_{i}}}\right\|: i=1, \ldots, r\right\}^{-1}$ and $s \in \mathbb{N}$ such that

$$
\left\|T_{n_{k_{s}}} u-x\right\|<\frac{\varepsilon}{2 \max \left\{\left\|T_{n_{k_{1}}}\right\|, \ldots,\left\|T_{n_{k_{r}}}\right\|\right\}} .
$$

Set $x_{i}=T_{n_{k_{i}}} u(i=1, \ldots, r)$. Then $x_{i} \in B_{X}$ and

$$
\left\|T_{n_{k_{s}}} x_{i}-y_{i}\right\|=\left\|T_{n_{k_{s}}} T_{n_{k_{i}}} u-y_{i}\right\| \leq\left\|T_{n_{k_{i}}}\left(T_{n_{k_{s}}} u-x\right)\right\|+\left\|T_{n_{k_{i}}} x-y_{i}\right\|<\varepsilon
$$

for all $i=1, \ldots, r$.

(ii) $\Rightarrow$ (iii). Clear.

(iii) $\Rightarrow$ (iv). Let $U, V, W \subset X$ be nonempty open sets, $0 \in W$. Let $\left(n_{k}\right)$ be a sequence of positive integers such that each subsequence of $\left(T_{n_{k}}\right)$ is hypercyclic. Let $x$ be a hypercyclic vector for $\left(T_{n_{k}}\right)$. Since each nonzero multiple of $x$ is also hypercyclic, we can assume that $x \in W$. Consider the subsequence $\left(T_{n_{k}}\right)_{k \in F}$ where $F=\left\{k: T_{n_{k}} x \in V\right\}$. Consequently, each $k \in F$ satisfies $T_{n_{k}} W \cap V \neq \emptyset$.

Let $y$ be a vector hypercyclic for this subsequence. Thus there exists $k_{0} \in F$ such that $T_{n_{k_{0}}} y \in U$. Moreover, we can choose increasing numbers $k_{i} \in F$ such that $T_{n_{k_{i}}} y \rightarrow 0(i \rightarrow \infty)$. Thus

$$
\lim _{i \rightarrow \infty} T_{n_{k_{i}}} T_{n_{k_{0}}} y=\lim _{i \rightarrow \infty} T_{n_{k_{0}}} T_{n_{k_{i}}} y=0
$$

and there is an $i$ with $T_{n_{k_{i}}} T_{n_{k_{0}}} y \in W$. Hence $T_{n_{k_{i}}} U \cap W \neq \emptyset$.

(iv) $\Rightarrow$ (i). By Proposition 6, the sequence $\left(T_{n}\right)$ has a dense subset of hypercyclic vectors.

Let $U_{1}, \ldots, U_{r}, V, W \subset X$ be nonempty open subsets, $0 \in W$. Let $x$ be a hypercyclic vector for the sequence $\left(T_{n}\right)$. Find $n_{1}, \ldots, n_{r} \in \mathbb{N}$ such that $T_{n_{i}} x \in U_{i}(i=1, \ldots, r)$. Let $\varepsilon>0$ satisfy $\left\{y:\left\|y-T_{n_{i}} x\right\|<\varepsilon\right\} \subset U_{i}$ $(i=1, \ldots, r)$ and $\{y:\|y\|<\varepsilon\} \subset W$. By assumption, there are $x^{\prime} \in X$ and $n_{0} \in \mathbb{N}$ such that $\left\|x^{\prime}-x\right\|<\varepsilon \max \left\{\left\|T_{n_{1}}\right\|, \ldots,\left\|T_{n_{r}}\right\|\right\}^{-1},\left\|T_{n_{0}} x^{\prime}\right\|<$ $\varepsilon \max \left\{\left\|T_{n_{1}}\right\|, \ldots,\left\|T_{n_{r}}\right\|\right\}^{-1}$ and $T_{n_{0}} W \cap V \neq \emptyset$. Then $\left\|T_{n_{i}} x^{\prime}-T_{n_{i}} x\right\| \leq$ 
$\left\|T_{n_{i}}\right\| \cdot\left\|x^{\prime}-x\right\|<\varepsilon$, and so $T_{n_{i}} x^{\prime} \in U_{i}(i=1, \ldots, r)$. Further $\left\|T_{n_{0}} T_{n_{i}} x^{\prime}\right\|=$ $\left\|T_{n_{i}} T_{n_{0}} x^{\prime}\right\| \leq\left\|T_{n_{i}}\right\| \cdot\left\|T_{n_{0}} x^{\prime}\right\|<\varepsilon$, and so $T_{n_{0}} T_{n_{i}} x^{\prime} \in W$.

Thus for commuting operators $T_{n}: X \rightarrow X$ we have the following situation:

$$
(\mathrm{HC}) \rightleftarrows(\mathrm{C}) \rightleftarrows(\text { dense hc) } \rightleftarrows(\mathrm{hc})
$$

A sequence $\left(T_{n}\right)$ of commuting operators satisfying condition $(\mathrm{C})$ but not $(\mathrm{HC})$ was given in Example 7.

An example of commuting operators with a dense set of hypercyclic vectors but not satisfying condition $(\mathrm{C})$ is the space $X=\mathbb{C}$ and $T_{n}(\lambda)=$ $r_{n} \lambda(\lambda \in \mathbb{C})$ where $\left(r_{n}\right)$ is a dense sequence in $\mathbb{C}$.

The existence of a hypercyclic sequence of commuting operators with a nondense set of hypercyclic vectors is an open problem:

Problem 13. Let $\left(T_{n}\right)$ be a hypercyclic sequence of mutually commuting operators acting on a Banach space $X$. Is the set of all vectors hypercyclic for $\left(T_{n}\right)$ dense in $X$ ?

IV. Commuting chains of operators. The most important case of a sequence of operators is the sequence $\left(T^{n}\right)$ of powers of a fixed operator $T \in B(X)$. Of importance are also sequences of the form $\left(\lambda_{n} T^{n}\right)$ where $T \in B(X)$ and $\lambda_{n}$ are nonzero complex numbers. Hypercyclicity of these sequences is closely connected with the supercyclicity of the operator $T$. Indeed, an operator $T \in B(X)$ is supercyclic (i.e., there exists $x \in X$ such that the set $\left\{\lambda T^{n} x: \lambda \in \mathbb{C}, n \geq 0\right\}$ is dense) if and only if there are complex numbers $\lambda_{n}$ such that the sequence $\left(\lambda_{n} T^{n}\right)$ is hypercyclic. In this way problems concerning the supercyclicity of operators reduce to problems concerning hypercyclicity of sequences of operators.

It turns out that the most important property of sequences $\left(T^{n}\right)$ or $\left(\lambda_{n} T^{n}\right)$ is that they form a chain of commuting operators. We call a sequence $\left(T_{n}\right) \subset B(X)$ a chain of commuting operators if there are mutually commuting operators $S_{j} \in B(X)$ such that $T_{n}=S_{1} \cdots S_{n}$ for all $n$.

For chains of commuting operators the situation is even simpler. A hypercyclic chain always has a dense subset of hypercyclic vectors and condition (C) is equivalent to $(\mathrm{HC})$.

Proposition 14. Let $S_{j} \in B(X)(j \in \mathbb{N})$ be mutually commuting operators and $T_{n}=S_{1} \cdots S_{n}$. Suppose that the sequence $\left(T_{n}\right)$ is hypercyclic. Then there exists a dense subset of vectors hypercyclic for $\left(T_{n}\right)$.

Proof. Let $x \in X$ be a vector hypercyclic for $\left(T_{n}\right)$. Clearly $T_{1} X \supset T_{2} X$ $\supset \cdots$, and so $T_{n}$ has dense range for all $n$. We show that $T_{j} x$ is hypercyclic for all $j$. We have $\left\{T_{n} T_{j} x: n \in \mathbb{N}\right\}^{-} \supset T_{j}\left\{T_{n} x: n \in \mathbb{N}\right\}^{-}=T_{j} X$, which is 
a dense subset of $X$. Hence $T_{j} x$ is hypercyclic and the sequence $\left(T_{n}\right)$ has a dense subset of hypercyclic vectors.

Theorem 15. Let $S_{j}: X \rightarrow X(j \in \mathbb{N})$ be mutually commuting operators and let $T_{n}=S_{1} \cdots S_{n}$. Suppose that the sequence $\left(T_{n}\right)$ satisfies condition $\left(\mathrm{C}_{\text {fin }}\right)$. Then it satisfies $(\mathrm{HC})$.

Proof. Since any subsequence of $\left(T_{n}\right)$ is again a chain of commuting operators, without loss of generality we can assume that $\left(T_{n}\right)$ satisfies condition $\left(\mathrm{C}_{\text {fin }}\right)$ for the whole sequence $\left(T_{n}\right)$, i.e., $T^{n} x \rightarrow 0$ for all $x$ in a dense subset of $X$.

Note first that for all $k, j \in \mathbb{N}$ we have

$$
\overline{\bigcup_{n>k}\left(S_{k+1} \cdots S_{n} B_{X}\right)^{j}}=Y^{j} \text {. }
$$

Indeed, we have $T_{k} B_{X} \subset\left\|T_{k}\right\| B_{X}$, and so

$$
\bigcup_{n>k}\left(S_{k+1} \cdots S_{n} B_{X}\right)^{j} \supset\left\|T_{k}\right\|^{-1} \bigcup_{n>k}\left(S_{1} \cdots S_{n} B_{X}\right)^{j}=\left\|T_{k}\right\|^{-1} \bigcup_{n>k}\left(T_{n} B_{X}\right)^{j},
$$

which is dense in $Y^{j}$.

Let $\left(x_{k}\right)$ be a sequence dense in $X$. By induction on $j$ we construct an increasing sequence $n_{j}$ and vectors $u_{k, j} \in X(k, j \in \mathbb{N}, j \geq k)$. Set formally $n_{0}=0$ and $u_{k, k}=x_{k}$.

Let $j \geq 2$ and suppose that $n_{j-1}$ and $u_{k, j-1} \in X(k \in \mathbb{N})$ have already been constructed. By (1), we can find $n_{j}>n_{j-1}$ and vectors $u_{k, j} \in X$ $(k=1, \ldots, j-1)$ such that

$$
\left\|S_{n_{j-1}+1} \cdots S_{n_{j}} u_{k, j}-u_{k, j-1}\right\|<\frac{1}{2^{k+j} \prod_{i \leq n_{j-1}} \max \left\{1,\left\|S_{i}\right\|\right\}}
$$

and $\left\|u_{k, j}\right\|<1 / 2^{k+j}$, which completes the construction.

Write for short $R_{j}=S_{n_{j-1}+1} \cdots S_{n_{j}}$. Then

$$
\left\|R_{j} u_{k, j}-u_{k, j-1}\right\|<\frac{1}{2^{k+j} \prod_{i \leq j-1} \max \left\{1,\left\|R_{i}\right\|\right\}}
$$

for all $k, j$, and $\left\|u_{k, j}\right\|<2^{-(k+j)}(k<j)$.

For fixed $k, j \in \mathbb{N}$ consider the sequence $\left(R_{j+1} \cdots R_{m} u_{k, m}\right)_{m=j}^{\infty}$. Since

$$
\begin{aligned}
& \left\|R_{j+1} \cdots R_{m+1} u_{k, m+1}-R_{j+1} \cdots R_{m} u_{k, m}\right\| \\
& \leq\left\|R_{j+1} \cdots R_{m}\right\| \cdot\left\|R_{m+1} u_{k, m+1}-u_{k, m}\right\| \leq 1 / 2^{k+m+1},
\end{aligned}
$$

the sequence $\left(R_{j+1} \cdots R_{m} u_{k, m}\right)_{m=j}^{\infty}$ is Cauchy. Denote by $v_{k, j}$ its limit. For all $k, j$ we have

$$
R_{j+1} v_{k, j+1}=\lim _{m \rightarrow \infty} R_{j+1} R_{j+2} \cdots R_{m} u_{k, m}=v_{k, j} .
$$


In particular, $T_{n_{j}} v_{k, j}=R_{1} \cdots R_{j} v_{k, j}=v_{k, 0}$ for all $k, j$. Furthermore,

$$
\begin{aligned}
\left\|v_{k, 0}-x_{k}\right\| & =\lim _{m \rightarrow \infty}\left\|R_{1} \cdots R_{m} u_{k, m}-u_{k, k}\right\| \\
& \leq \sum_{m=k}^{\infty}\left\|R_{1} \cdots R_{m+1} u_{k, m+1}-R_{1} \cdots R_{m} u_{k, m}\right\| \\
& \leq \sum_{m=0}^{\infty} \frac{1}{2^{k+m+1}}=\frac{1}{2^{k}},
\end{aligned}
$$

and so the sequence $\left(v_{k, 0}\right)$ is dense in $X$.

Finally, for $j>k$ we have

$$
\begin{aligned}
\left\|v_{k, j}\right\| & =\lim _{m \rightarrow \infty}\left\|R_{j+1} \cdots R_{m} u_{k, m}\right\| \\
& \leq\left\|u_{k, j}\right\|+\sum_{m=j}^{\infty}\left\|R_{j+1} \cdots R_{m+1} u_{k, m+1}-R_{j+1} \cdots R_{m} u_{k, m}\right\| \\
& \leq\left\|u_{k, j}\right\|+\sum_{m=j}^{\infty}\left\|R_{1} \cdots R_{m}\right\| \cdot\left\|R_{m+1} u_{k, m+1}-u_{k, m}\right\| \\
& \leq \frac{1}{2^{k+j}}+\sum_{m=j}^{\infty} \frac{1}{2^{k+m+1}}=\frac{1}{2^{k+j-1}},
\end{aligned}
$$

and so $\lim _{j \rightarrow \infty}\left\|v_{k, j}\right\|=0$. Hence the sequence $\left(T_{n}\right)$ satisfies condition (HC) for the sequence $\left(n_{j}\right)$ and the dense set $\left\{v_{k, 0}: k \in \mathbb{N}\right\}$.

Corollary 16. Let $T \in B(X)$ and let $\left(\lambda_{n}\right)$ be a sequence of complex numbers. Then all the conditions $(\mathrm{C}),\left(\mathrm{C}_{\mathrm{fin}}\right),(\mathrm{HC}),($ her hc) and $(4 \mathrm{nbhd})$ are equivalent for the sequence $\left(\lambda_{n} T^{n}\right)$.

If $\left(\lambda_{n} T^{n}\right)$ is hypercyclic then there is a dense subset of hypercyclic vectors.

PROBLEM 17. Is there a chain of commuting operators (and in particular a sequence of the form $\left(T^{n}\right)$ ) which is hypercyclic but does not satisfy the hypercyclicity criterion (or any equivalent conditions)?

V. Subspaces of hypercyclic vectors. In this section we study the existence of a dense (closed infinite-dimensional, respectively) subspace consisting of hypercyclic vectors.

In the case of a hypercyclic sequence $\left(T^{n}\right)$ where $T \in B(X)$ is a fixed operator it is known that there is always a dense subspace consisting of hypercyclic vectors. The proof, however, uses special properties of the sequence $\left(T^{n}\right)$.

Our first result gives the existence of a dense subspace consisting of hypercyclic vectors for any sequence $\left(T_{n}\right) \subset B(X, Y)$ satisfying $\mathrm{C}_{\mathrm{fin}}$. 
THEOREM 18. Let $\left(T_{n}\right) \subset B(X, Y)$ be a sequence of operators satisfying condition $\left(\mathrm{C}_{\mathrm{fin}}\right)$. Then there exists a dense subspace $X_{1} \subset X$ such that each nonzero vector in $X_{1}$ is hypercyclic for $\left(T_{n}\right)$.

Proof. Let $Z$ be any separable infinite-dimensional Banach space. Let $x \in X, x \neq 0$ and $\varepsilon>0$. Set $\mathcal{M}=\{V \in \overline{F(Z, X)}$ : $\operatorname{dist}\{x, V Z\}<\varepsilon\}$. Clearly $\mathcal{M}$ is open. We show that it is dense in $\overline{F(Z, X)}$.

Let $W \in \overline{F(Z, X)}$ and $\delta>0$. Then there exists a finite rank operator $W_{1}: Z \rightarrow X$ such that $\left\|W-W_{1}\right\|<\delta / 2$. Let $z \in \operatorname{ker} W_{1}$ and $z^{*} \in Z^{*}$ satisfy $\left\langle z, z^{*}\right\rangle=1$. Set

$$
W_{2}=W_{1}+\frac{\delta \cdot\left(z^{*} \otimes x\right)}{2\|x\| \cdot\left\|z^{*}\right\|}
$$

Then

$$
\left\|W-W_{2}\right\| \leq\left\|W-W_{1}\right\|+\left\|W_{1}-W_{2}\right\|<\delta \quad \text { and } \quad W_{2} z=\frac{\delta x}{2\|x\| \cdot\left\|z^{*}\right\|} .
$$

Thus $W_{2} \in \mathcal{M}$ and $\mathcal{M}$ is dense in $\overline{F(Z, X)}$.

Let $\left(x_{k}\right) \subset X$ be a dense sequence of nonzero vectors. Clearly $V \in$ $\overline{F(Z, X)}$ has dense range if and only if $\operatorname{dist}\left\{x_{k}, V Z\right\}<1 / k$ for all $k$. By the Baire category theorem, the set of all operators in $\overline{F(Z, X)}$ with dense range is residual.

By Theorem 5 , the operators $L_{T_{n}}: \overline{F(Z, X)} \rightarrow \overline{F(Z, Y)}$ satisfy condition (C), and so there is a residual set of vectors hypercyclic for $\left(L_{T_{n}}\right)$. Thus there exists an operator $V \in \overline{F(Z, X)}$ with dense range such that $V$ is hypercyclic for $\left(L_{T_{n}}\right)$.

It is easy to see that each nonzero vector in the range $V Z$ is hypercyclic for the sequence $\left(T_{n}\right)$. This completes the proof.

Next we study the existence of a closed infinite-dimensional subspace consisting of hypercyclic vectors for a sequence $\left(T_{n}\right) \subset B(X, Y)$. Such a subspace is known to exist (under a natural additional assumption) if $\left(T_{n}\right)$ is hereditarily hypercyclic. We prove it now for sequences satisfying the more practical condition (C). Moreover, the proof is essentially simplified.

Note that a particularly simple argument is available in the case of a sequence $\left(T^{n}\right)$ satisfying the hypercyclicity criterion (HC) (see [ChT]).

We say for short that a subspace $X_{1} \subset X$ is a hypercyclic subspace for a sequence $\left(T_{n}\right) \subset B(X, Y)$ if each nonzero vector in $X_{1}$ is hypercyclic for $\left(T_{n}\right)$.

Theorem 19 (cf. $[\mathrm{Mo}])$. Let $\left(T_{n}\right) \subset B(X, Y)$ be a sequence of operators. Suppose that $\left(n_{k}\right)$ is an increasing sequence of positive integers such that

(i) there exists a dense subset $X_{0} \subset X$ such that $\lim _{k \rightarrow \infty} T_{n_{k}} x=0$

(ii) $\frac{\left(x \in X_{0}\right) ;}{\bigcup_{k \in \mathbb{N}} T_{n_{k}} B_{X}}=Y$; 
(iii) there exists a closed infinite-dimensional subspace $X_{1} \subset X$ with the property that $\lim _{k \rightarrow \infty} T_{n_{k}} x=0 \quad\left(x \in X_{1}\right)$.

Then there exists a closed infinite-dimensional hypercyclic subspace for $\left(T_{n}\right)$.

Proof. Without loss of generality we can assume that $\lim _{n \rightarrow \infty} T_{n} x=0$ for all $x \in X_{0} \cup X_{1}$. Let $\left\{e_{1}, e_{2}, \ldots\right\}$ be a normalized basic sequence in $X_{1}$. Let $K$ be the corresponding basic constant and let $\varepsilon<1 / 2 K$. Let $\left(y_{k}\right)$ be a dense sequence in $Y$. Let $\prec$ be an order on $\mathbb{N} \times(\mathbb{N} \cup\{0\})$ defined by $(i, j) \prec\left(i^{\prime}, j^{\prime}\right)$ if either $i+j<i^{\prime}+j^{\prime}$, or $i+j=i^{\prime}+j^{\prime}$ and $i<i^{\prime}$.

Set $z_{i, 0}=e_{i}(i=1,2, \ldots)$. By induction with respect to the order $\prec$ we construct vectors $z_{i, j} \in X_{0}(i, j \in \mathbb{N})$ and an increasing sequence $n_{i, j} \subset \mathbb{N}$.

Let $(i, j) \in \mathbb{N} \times \mathbb{N}$ and suppose that $z_{i^{\prime}, j^{\prime}} \in X_{0}$ and $n_{i^{\prime}, j^{\prime}} \in \mathbb{N}$ have already been constructed for all $\left(i^{\prime}, j^{\prime}\right) \prec(i, j)$. By definition, there exist $n_{i, j}>\max \left\{n_{i^{\prime}, j^{\prime}}:\left(i^{\prime}, j^{\prime}\right) \prec(i, j)\right\}$ and $z_{i, j} \in X_{0}$ such that

$$
\begin{gathered}
\left\|T_{n_{i, j}} z_{i^{\prime}, j^{\prime}}\right\|<\frac{\varepsilon}{2^{i^{\prime}+j^{\prime}+i}} \quad\left(\left(i^{\prime}, j^{\prime}\right) \prec(i, j)\right), \quad\left\|T_{n_{i, j}} z_{i, j}-y_{i}\right\|<\frac{\varepsilon}{2^{2 i+j}}, \\
\left\|z_{i, j}\right\|<\frac{\varepsilon}{2^{i+j} \max \left\{1,2^{i^{\prime}}\left\|T_{n_{i^{\prime}, j^{\prime}}}\right\|:\left(i^{\prime}, j^{\prime}\right) \prec(i, j)\right\}} .
\end{gathered}
$$

In this way, the vectors $z_{i, j} \in X_{0}$ and numbers $n_{i, j}$ are inductively constructed.

Set $z_{i}=\sum_{j=0}^{\infty} z_{i, j}(i \in \mathbb{N})$. Then

$$
\left\|z_{i}-e_{i}\right\| \leq \sum_{j=1}^{\infty}\left\|z_{i, j}\right\|<\sum_{j=1}^{\infty} \frac{\varepsilon}{2^{i+j}}=\frac{\varepsilon}{2^{i}} .
$$

Hence $\sum_{i=1}^{\infty}\left\|z_{i}-e_{i}\right\|<\sum_{i=1}^{\infty} \varepsilon / 2^{i}=\varepsilon$, and so $\left(z_{i}\right)$ is a basic sequence.

Let $M=\bigvee\left\{z_{i}: i=1,2, \ldots\right\}$. Let $z \in M$ be any nonzero vector. Then $z=\sum_{i=1}^{\infty} \alpha_{i} z_{i}$ for some complex coefficients $\alpha_{i}$. We show that $z$ is hypercyclic for $\left(T_{n}\right)$.

Fix $k \in \mathbb{N}$ with $\alpha_{k} \neq 0$. Since every nonzero scalar multiple of a hypercyclic vector is also hypercyclic, we can assume that $\alpha_{k}=1$. Then

$$
\begin{aligned}
\left\|T_{n_{k}, r} z-y_{r}\right\| & \leq \sum_{i \neq k}\left|\alpha_{i}\right| \cdot\left\|T_{n_{k}, r} z_{i}\right\|+\left\|T_{n_{k, r}} z_{k}-y_{r}\right\| \\
& \leq \sum_{i \neq k} \sum_{j=0}^{\infty}\left|\alpha_{i}\right| \cdot\left\|T_{n_{k, r}} z_{i, j}\right\|+\sum_{j \neq r}\left\|T_{n_{k, r}} z_{k, j}\right\|+\left\|T_{n_{k, r}} z_{k, r}-y_{r}\right\| \\
& \leq \sum_{(i, j) \prec(k, r)} \max \left\{\left|\alpha_{i}\right|: i \in \mathbb{N}\right\} \cdot\left\|T_{n_{k, r}} z_{i, j}\right\|
\end{aligned}
$$




$$
\begin{aligned}
& +\sum_{(k, r) \prec(i, j)} \max \left\{\left|\alpha_{i}\right|: i \in \mathbb{N}\right\} \cdot\left\|T_{n_{k, r}} z_{i, j}\right\|+\left\|T_{n_{k, r}} z_{k, r}-y_{r}\right\| \\
< & \sum_{(i, j) \prec(k, r)} \max \left\{\left|\alpha_{i}\right|: i \in \mathbb{N}\right\} \cdot \frac{\varepsilon}{2^{i+j+k}} \\
& +\sum_{(k, r) \prec(i, j)} \max \left\{\left|\alpha_{i}\right|: i \in \mathbb{N}\right\} \cdot \frac{\varepsilon}{2^{i+j+k}}+\frac{\varepsilon}{2^{2 k+r}} \\
\leq & \sum_{i=1}^{\infty} \sum_{j=0}^{\infty} \max \left\{\left|\alpha_{i}\right|: i \in \mathbb{N}\right\} \cdot \frac{\varepsilon}{2^{i+j+k}} \leq \frac{K \varepsilon}{2^{k-1}} .
\end{aligned}
$$

Hence $z$ is hypercyclic for $\left(T_{n}\right)$.

Theorem 20. Let $\left(T_{n}\right) \subset B(X, Y)$ be a sequence of operators satisfying condition (C) for a subsequence $\left(n_{k}\right)$. Suppose that there are infinitedimensional subspaces $M_{1}, M_{2}, \ldots$ such that $X \supset M_{1} \supset M_{2} \supset \cdots$ and $\sup _{k}\left\|T_{n_{k}} \mid M_{k}\right\|<\infty$. Then there exists a closed infinite-dimensional hypercyclic subspace for $\left(T_{n}\right)$.

Proof. Without loss of generality we can assume that $T_{n} x \rightarrow 0$ for all $x$ in a dense subset $X_{0} \subset X$. It is sufficient to construct a closed infinitedimensional subspace $X_{1} \subset X$ such that $T_{n} x \rightarrow 0\left(x \in X_{1}\right)$.

We can find a basic sequence $\left(x_{n}\right)$ such that $x_{i} \in M_{i}$ for all $i$. Let $K$ be the basic constant of this sequence. Let $\varepsilon<1 / 2 K$ be a positive number. For each $n$ find $e_{n} \in X_{0}$ such that

$$
\left\|x_{n}-e_{n}\right\|<\frac{\varepsilon}{2^{n} \max \left\{1,\|T\|, \ldots,\left\|T^{n}\right\|\right\}} .
$$

Clearly $\left(e_{n}\right)$ is a basic sequence with basic constant $\leq 2 K$. Let $\left(y_{n}\right)$ be a dense sequence in $Y$. Choose a subsequence $\left(e_{n_{k}}\right)$ such that $\left\|T_{n_{k}} e_{n_{i}}\right\|<$ $2^{-(k+i)}(i<k)$ and $\operatorname{dist}\left\{y_{k}, T_{n_{k}} B_{X}\right\}<2^{-k}$. Set $X_{1}=\bigvee\left\{e_{n_{k}}: k \in \mathbb{N}\right\}$. Let $e \in X_{1}$ be an arbitrary vector. We can write $e=\sum_{i=1}^{\infty} \alpha_{i} e_{n_{i}}$ for some complex coefficients $\alpha_{i}$. We have

$$
\begin{aligned}
\left\|T_{n_{k}} e\right\| & \leq \sum_{i=1}^{k-1}\left\|T_{n_{k}} \alpha_{i} e_{n_{i}}\right\|+\left\|\sum_{i=k}^{\infty} T_{n_{k}} \alpha_{i} x_{n_{i}}\right\|+\sum_{i=k}^{\infty}\left\|T_{n_{k}} \alpha_{i}\left(e_{n_{i}}-x_{n_{i}}\right)\right\| \\
& \leq 2 K \sum_{i=1}^{k-1} \frac{1}{2^{i+k}}+\sup _{n}\left\|T_{n} \mid M_{n}\right\| \cdot\left\|\sum_{i=k}^{\infty} \alpha_{i} x_{n_{i}}\right\|+2 K \sum_{i=k}^{\infty} \frac{\varepsilon}{2^{i}} \\
& \leq \frac{K}{2^{k-1}}+\sup _{n}\left\|T_{n} \mid M_{n}\right\| \cdot\left\|\sum_{i=k}^{\infty} \alpha_{i} x_{n_{i}}\right\|+\frac{K \varepsilon}{2^{k-2}} \rightarrow 0
\end{aligned}
$$

as $k \rightarrow \infty$. Further $\overline{\bigcup_{j} T_{n_{j}} B_{X}}=Y$, and so there is a closed infinitedimensional subspace consisting of hypercyclic vectors for $\left(T_{n}\right)$. 
We now give a negative result - a condition implying that there is no closed infinite-dimensional subspace consisting of hypercyclic vectors.

Recall the quantity $j_{\mu}(T)=\sup \{j(T \mid M): M \subset X, \operatorname{codim} M<\infty\}$, where $j$ denotes the minimum modulus, $j(S)=\inf \{\|S x\|:\|x\| \leq 1\}$. The number $j_{\mu}(T)$ can be called the essential minimum modulus of $T$.

Lemma 21. Let $T_{1}, \ldots, T_{k} \in B(X, Y)$ and let $X_{1} \subset X$ be a closed infinite-dimensional subspace. Let $\varepsilon>0$. Then there exists $x \in X_{1}$ of norm one such that $\left\|T_{i} x\right\|>j_{\mu}\left(T_{i}\right)-\varepsilon(i=1, \ldots, k)$.

Proof. For $i=1, \ldots, k$ there is a subspace $M_{i} \subset X$ of finite codimension such that $j\left(T_{i} \mid M_{i}\right)>j_{\mu}\left(T_{i}\right)-\varepsilon$. Let $x$ be any vector of norm one in $X_{1} \cap$ $\bigcap_{i=1}^{k} M_{i}$. Then

$$
\left\|T_{i} x\right\| \geq j\left(T_{i} \mid M_{i}\right)>j_{\mu}\left(T_{i}\right)-\varepsilon
$$

for all $i=1, \ldots, k$.

Theorem 22. Let $X, Y$ be Banach spaces, let $\left(T_{n}\right) \subset B(X, Y)(n=$ $1,2, \ldots)$, let $\left(a_{n}\right)$ be a sequence of positive numbers such that $\lim _{i \rightarrow \infty} a_{i}=0$ and let $X_{1} \subset X$ be a closed infinite-dimensional subspace. Let $\delta>0$. Then there exists a vector $x \in X_{1}$ with $\|x\| \leq \sup _{i} a_{i}+\delta$ and $\left\|T_{n} x\right\| \geq a_{n} j_{\mu}\left(T_{n}\right)$ for all $n \in \mathbb{N}$.

Moreover, there is a subset $X_{2}$ dense in $X_{1}$ with the property that for each $x \in X_{2}$ there exists $n_{0}$ such that $\left\|T_{n} x\right\| \geq a_{n} j_{\mu}\left(T_{n}\right)\left(n \geq n_{0}\right)$.

Proof. Without loss of generality we can assume that $a_{1} \geq a_{2} \geq \cdots$. Let $\varepsilon>0$ satisfy $(1-\varepsilon)^{2}\left(a_{1}+\delta / 2\right)>a_{1}$. Find numbers $r_{0}<r_{1}<\cdots$ such that $a_{r_{k}}<(1-\varepsilon)^{3} \delta / 2^{k+3}$ for all $k$. Find $x_{0} \in X_{1}$ such that $\left\|x_{0}\right\|=a_{1}+\delta / 2$ and $\left\|T_{n} x_{0}\right\|>(1-\varepsilon)\left(a_{1}+\delta / 2\right) j_{\mu}\left(T_{n}\right)\left(n \leq r_{0}\right)$.

Let $k \geq 0$ and suppose that $x_{0}, \ldots, x_{k}$ have already been constructed. Let $E_{k}=\bigvee\left\{T_{n} x_{i}: 0 \leq i \leq k, 1 \leq n \leq r_{k+1}\right\}$. Let $M_{k}$ be a subspace of $X$ of finite codimension such that

$$
\|e+m\| \geq(1-\varepsilon) \max \{\|e\|,\|m\| / 2\} \quad\left(e \in E_{k}, m \in M_{k}\right)
$$

(see $[\mathrm{M}]$ ). Since the space $L_{k}=\bigcap_{i=1}^{k} \bigcap_{n=1}^{r_{k+1}} T_{n}^{-1} M_{i}<\infty$ is of finite codimension, we can choose $x_{k+1} \in X_{1} \cap L_{k}$ such that $\left\|x_{k+1}\right\|=\delta 2^{-(k+2)}$ and

$$
\left\|T_{n} x_{k+1}\right\| \geq(1-\varepsilon) \delta 2^{-(k+2)} j_{\mu}\left(T_{n}\right) \quad\left(1 \leq n \leq r_{k+1}\right) .
$$

Set $x=\sum_{i=0}^{\infty} x_{i}$. Then $x \in X_{1}$ and

$$
\|x\| \leq \sum_{i=0}^{\infty}\left\|x_{i}\right\| \leq a_{1}+\delta / 2+\sum_{i=1}^{\infty} \delta 2^{-(i+1)}=a_{1}+\delta .
$$

For $n=1, \ldots, r_{0}$ we have

$$
\left\|T_{n} x\right\|=\left\|T_{n} x_{0}+\sum_{i=1}^{\infty} T_{n} x_{i}\right\| \geq(1-\varepsilon)\left\|T_{n} x_{0}\right\|>a_{1} j_{\mu}\left(T_{n}\right) \geq a_{n} j_{\mu}\left(T_{n}\right) .
$$


Let $k \geq 0$ and $r_{k}<n \leq r_{k+1}$. Then

$$
\begin{aligned}
\left\|T_{n} x\right\| & =\left\|\sum_{i=0}^{\infty} T_{n} x_{i}\right\| \geq(1-\varepsilon)\left\|\sum_{i=0}^{k+1} T_{n} x_{i}\right\| \\
& \geq \frac{(1-\varepsilon)^{2}}{2}\left\|T_{n} x_{k+1}\right\| \geq \frac{(1-\varepsilon)^{3}}{2} \cdot \frac{\delta}{2^{k+2}} j_{\mu}\left(T_{n}\right) \geq a_{n} j_{\mu}\left(T_{n}\right) .
\end{aligned}
$$

Thus $\left\|T_{n} x\right\| \geq a_{n} j_{\mu}\left(T_{n}\right)$ for all $n \in \mathbb{N}$.

To show the second statement, let $u \in X_{1}$ and $\varepsilon>0$. Find $n_{0}$ such that $a_{n}<\varepsilon$ for all $n \geq n_{0}$. As in the first part, taking $x_{0}=u$, construct a vector $x \in X_{1}$ with $\|x-u\| \leq \varepsilon$ and $\left\|T_{n} x\right\| \geq a_{n} j_{\mu}\left(T_{n}\right)\left(n \geq n_{0}\right)$.

Corollary 23. Let $X, Y$ be Banach spaces and let $\left(T_{n}\right) \subset B(X, Y)$ be a sequence of operators satisfying $\lim _{n \rightarrow \infty} j_{\mu}\left(T_{n}\right)=\infty$. Then there is no closed infinite-dimensional hypercyclic subspace for $\left(T_{n}\right)$.

Proof. Let $M$ be a closed infinite-dimensional subspace of $X$. By the previous result for the numbers $\alpha_{n}=\left(j_{\mu}\left(T_{n}\right)\right)^{-1 / 2}$, there exists $x \in M$ such that $\left\|T_{n} x\right\| \rightarrow \infty$. Therefore $x$ is not hypercyclic for $\left(T_{n}\right)$.

We apply the previous results to the sequences of the form $\left(\lambda_{n} T^{n}\right)$ where $T \in B(X)$ and $\lambda_{n}$ are complex numbers. Denote by $\sigma_{\mathrm{e}}(T)=\{\lambda \in \mathbb{C}: T-\lambda$ is not Fredholm $\}$ the essential spectrum of $T$.

Corollary 24. Let $T \in B(X)$ be an operator and let $\left(\lambda_{n}\right)$ be a sequence of complex numbers. Suppose that $\left(\lambda_{n} T^{n}\right)$ satisfies condition $(\mathrm{C})$ and $\sup _{n}\left|\lambda_{n}\right| d^{n}<\infty$ where $d=\operatorname{dist}\left\{0, \sigma_{\mathrm{e}}(T)\right\}$. Then there exists a closed infinite-dimensional hypercyclic subspace for $\left(\lambda_{n} T^{n}\right)$.

Proof. Since $\left(\lambda_{n} T^{n}\right)$ satisfies condition (C), the range of $T$ is dense. Without loss of generality we can assume that the numbers $\lambda_{n}$ are nonzero.

Choose $\lambda \in \sigma_{\mathrm{e}}(T)$ with $|\lambda|=d$. Thus $T-\lambda$ is not Fredholm. We show that $T-\lambda$ is not upper semi-Fredholm. This is clear if $d=0$ since the range of $T$ is dense. If $d>0$ then $\lambda \in \partial \sigma_{\mathrm{e}}(T)$ and $T-\lambda$ is not upper semi-Fredholm by $[\mathrm{HW}]$.

By [LS], there is a compact operator $K \in B(X)$ with $\operatorname{dim} \operatorname{ker}(T-\lambda-K)$ $=\infty$. Set $M_{0}=\operatorname{ker}(T-\lambda-K)$. For each $n$ we have $T^{n}=(T-K)^{n}+K_{n}$ for some compact operator $K_{n}$. Find subspaces $M_{n}^{\prime} \subset X$ of finite codimension such that $\left\|\left.K_{n}\left|M_{n}^{\prime} \| \leq\right| \lambda_{n}\right|^{-1}\right.$. Set $M_{n}=M_{0} \cap \bigcap_{i<n} M_{i}^{\prime}$. Then $M_{1} \supset M_{2}$ $\supset \cdots$ and $\operatorname{dim} M_{n}<\infty$. For $z \in M_{n}$ with $\|z\|=1$ we have

$$
\left\|\lambda_{n} T^{n} z\right\| \leq\left\|\lambda_{n}(T-K)^{n} z\right\|+\left\|\lambda_{n} K_{n}\right\| \leq\left|\lambda_{n} \lambda^{n}\right|+1=\left|\lambda_{n}\right| d^{n}+1,
$$

and so $\sup _{n}\left\|\lambda_{n} T^{n} \mid M_{n}\right\|<\infty$. The statement now follows from Theorem 20 . 
Corollary 25. Let $T: X \rightarrow X$ and suppose that $\left(\lambda_{n} T^{n}\right)$ satisfies $(\mathrm{C})$ and $T$ is not Fredholm. Then there is an infinite-dimensional closed hypercyclic subspace for $\left(\lambda_{n} T^{n}\right)$.

Proof. We have $d=\operatorname{dist}\left\{0, \sigma_{\mathrm{e}}(T)\right\}=0$, and so the statement follows from the previous corollary.

Corollary 26. Let $T \in B(X)$ and suppose that $\left(T^{n}\right)$ satisfies $(\mathrm{C})$. The following conditions are equivalent:

(i) there exists a closed infinite-dimensional hypercyclic subspace for $\left(T^{n}\right)$;

(ii) the essential spectrum of $T$ intersects the closed unit ball.

Proof. Write $d=\operatorname{dist}\left\{0, \sigma_{\mathrm{e}}(T)\right\}$.

(ii) $\Rightarrow$ (i). If $d \leq 1$ then Corollary 24 implies (i).

(i) $\Rightarrow$ (ii). Let $d>1$. Then $T$ is Fredholm. Recall the following standard construction from operator theory (see $[\mathrm{S}],[\mathrm{BHW}]$ ): let $\ell^{\infty}(X)$ be the space of all bounded sequences of elements of $X$; with the naturally defined algebraic operations and sup-norm it is a Banach space. Let $\widetilde{X}=\ell^{\infty}(X) / m(X)$ where $m(X)$ is the subspace of all precompact sequences. Let $\widetilde{T}: \widetilde{X} \rightarrow \widetilde{X}$ be the operator induced by $T$. It is well known that $\widetilde{T}$ is invertible and $\sigma(\widetilde{T})=\sigma_{\mathrm{e}}(T)$. By the spectral radius formula we have $d=\operatorname{dist}\{0, \sigma(\widetilde{T})\}=$ $r\left(\widetilde{T}^{-1}\right)^{-1}=\lim _{n \rightarrow \infty}\left\|\widetilde{T}^{-n}\right\|^{-1 / n}=\lim _{n \rightarrow \infty} j\left(\widetilde{T}^{n}\right)^{1 / n}$ where $r$ denotes the spectral radius. By $[\mathrm{F}]$ we find that $j_{\mu}\left(T^{n}\right) \leq 2 j\left(\widetilde{T}^{n}\right) \leq 4 j_{\mu}\left(T^{n}\right)$ for all $n$. Thus $1<d=\lim _{n \rightarrow \infty} j_{\mu}\left(T^{n}\right)^{1 / n}$ and $\lim _{n \rightarrow \infty} j_{\mu}\left(T^{n}\right)=\infty$.

Let $M$ be a closed infinite-dimensional subspace of $X$. By Theorem 22 for the numbers $\alpha_{n}=\left(j_{\mu}\left(T^{n}\right)\right)^{-1 / 2}$, there exists $x \in M$ such that $\lim _{n \rightarrow \infty}\left\|T^{n} x\right\|$ $=\infty$. Hence $x$ is not hypercyclic for $\left(T^{n}\right)$.

\section{References}

[Bea] B. Beauzamy, Un opérateur, sur l'espace de Hilbert, dont tous les polynômes sont hypercycliques, C. R. Acad. Sci. Paris Sér. I Math. 303 (1986), 923-925.

[B] L. Bernal-González, Densely hereditarily hypercyclic sequences and large hypercyclic manifolds, Proc. Amer. Math. Soc. 127 (1999), 3279-3285.

[BC] L. Bernal-González and M. C. Calderón-Moreno, Dense linear manifolds of monsters, J. Approx. Theory 119 (2002), 156-180.

[BG] L. Bernal-González and K.-G. Grosse-Erdmann, The hypercyclicity criterion for sequences of operators, Studia Math. 157 (2003), 17-32.

[BP] J. Bès and A. Peris, Hereditarily hypercyclic operators, J. Funct. Anal. 167 (1999), 94-112.

[BMP] J. Bonet, F. Martínez-Giménez and A. Peris, Universal and chaotic multipliers on spaces of operators, J. Math. Anal. Appl. 297 (2004), 599-611.

[Bo] P. S. Bourdon, Invariant manifolds of hypercyclic vectors, Proc. Amer. Math. Soc. 118 (1993), 845-847. 
[BHW] J. J. Buoni, R. Harte and T. Wickstead, Upper and lower Fredholm spectra, ibid. 66 (1977), 309-314.

[ChT] K. C. Chan and R. D. Taylor Jr., Hypercyclic subspaces of a Banach space, Integral Equations Operator Theory 41 (2001), 381-388.

[F] A. S. Fănshteĭn, Measures of noncompactness of linear operators and analogues of the minimum modulus for semi-Fredholm operators, in: Spectral Theory of Operators 6, Èlm, Baku, 1985, 182-195 (in Russian).

[G] G. Godefroy, Opérateurs ayant un sous-espace dense de vecteurs hypercycliques, Séminaire d'Initiation à l'Analyse, Publ. Math. Univ. Pierre et Marie Curie 94, Paris, 1989.

[GS] G. Godefroy and J. H. Shapiro, Operators with dense, invariant, cyclic vector manifolds, J. Funct. Anal. 98 (1991), 229-269.

[GLM] M. González, F. León-Saavedra and A. Montes-Rodríguez, Semi-Fredholm theory: hypercyclic and supercyclic subspaces, Proc. London Math. Soc. 81 (2000), 169-189.

[Gri] S. Grivaux, Construction of operators with prescribed behaviour, Arch. Math. (Basel) 81 (2003), 291-299.

[HW] R. Harte and T. Wickstead, Upper and lower Fredholm spectra II, Math. Z. 154 (1977), 253-256.

[He] D. A. Herrero, Limits of hypercyclic and supercyclic operators, J. Funct. Anal. 99 (1991), 179-190.

[K] C. Kitai, Invariant closed sets for linear operators, dissertation, Univ. of Toronto, Toronto, 1982.

[LS] A. Lebow and M. Schechter, Semigroups of operators and measures of noncompactness, J. Funct. Anal. 7 (1971), 1-26.

[LMo] F. León-Saavedra and A. Montes-Rodríguez, Spectral theory and hypercyclic subspaces, Trans. Amer. Math. Soc. 353 (2000), 247-267.

[Mo] A. Montes-Rodríguez, Banach spaces of hypercyclic vectors, Michigan Math. J. 43 (1996), 419-436.

[M] V. Müller, Local behaviour of the polynomial calculus of operators, J. Reine Angew. Math. 430 (1992), 61-68.

[S] B. N. Sadovskiǔ, Limit-compact and condensing operators, Uspekhi Mat. Nauk 27 (1972), no. 1, 81-146 (in Russian); English transl.: Russian Math. Surveys 27 (1972), no. $1,85-155$.

Departamento de Matemáticas

Mathematical Institute

Facultad de Ciencias

Universidad de Cádiz

Pol. Rio San Pedro S/N

1500 Puerto Real, Spain

Žitná 25

E-mail: fernando.leon@uca.es

Czech Academy of Sciences

11567 Praha 1, Czech Republic

E-mail: muller@math.cas.cz

Received June 21, 2003

Revised version March 2, 2006 